\title{
Rate-Constrained Coder Control and Comparison of Video Coding Standards
}

\author{
Thomas Wiegand, Heiko Schwarz, Anthony Joch, Faouzi Kossentini, Senior Member, IEEE, and \\ Gary J. Sullivan, Senior Member, IEEE
}

\begin{abstract}
A unified approach to the coder control of video coding standards such as MPEG-2, H.263, MPEG-4, and the draft video coding standard H.264/AVC is presented. The performance of the various standards is compared by means of PSNR and subjective testing results. The results indicate that $\mathrm{H.264/AVC}$ compliant encoders typically achieve essentially the same reproduction quality as encoders that are compliant with the previous standards while typically requiring $60 \%$ or less of the bit rate.
\end{abstract}

Index Terms-Coder control, Lagrangian, H.263, H.264/AVC, MPEG-2, MPEG-4, rate-constrained, standards, video.

\section{INTRODUCTION}

$\mathbf{T}$ HE specifications of most video coding standards including MPEG-2 Visual [1] , H.263 [2], MPEG-4 Visual [3] and H.264/AVC [4] provide only the bit-stream syntax and the decoding process in order to enable interoperability. The encoding process is left out of the scope to permit flexible implementations. However, the operational control of the source encoder is a key problem in video compression. For the encoding of a video source, many coding parameters such as macroblock modes, motion vectors, and transform coefficient levels have to be determined. The chosen values determine the rate-distortion efficiency of the produced bitstream of a given encoder.

In this paper, the operational control of MPEG-2, H.263, MPEG-4, and H.264/AVC encoders is optimized with respect to their rate-distortion efficiency using Lagrangian optimization techniques. The optimization is based on [5] and [6], where the encoder control for the ITU-T Recommendation H.263 [2] is addressed. The Lagrangian coder control as described in this paper was also integrated into the test models TMN-10 [7] and JM-2 [8] for the ITU-T Recommendation H.263 and H.264/AVC, respectively. The same Lagrangian coder control method was also applied to the MPEG-4 verification model VM-18 [9] and the MPEG-2 test model TM-5 [10]. In addition to achieving performance gains, the use of similar rate-dis-

Manuscript received December 12, 2001; revised May 10, 2003.

T. Wiegand and H. Schwarz are with the Fraunhofer-Institute for Telecommunications, Heinrich-Hertz Institute, 10587 Berlin, Germany (e-mail: wiegand@hhi.de; hschwarz@hhi.de)

A. Joch is with UB Video Inc., Vancouver, BC V6B 2R9, Canada (e-mail: anthony@ubvideo.com).

F. Kossentini is with the Department of Electrical and Computer Engineering, University of British Columbia, Vancouver, BC V6T 1Z4, Canada (e-mail: faouzi@ece.ubc.ca).

G. J. Sullivan is with the Microsoft Corporation, Redmond, WA 98052 USA (e-mail: garysull@microsoft.com).

Digital Object Identifier 10.1109/TCSVT.2003.815168 tortion optimization methods in all encoders allows a useful comparison between the encoders in terms of coding efficiency.

This paper is organized as follows. Section II gives an overview of the syntax features of MPEG-2 Video, H.263, MPEG-4 Visual, and H.264/AVC. The rate-distortion-optimized coder control is described in Section III, and experimental results are presented in Section IV.

\section{STANDARD SyntaX AND DECODERS}

All ITU-T and ISO/IEC JTC1 standards since H.261 [11] have in common that they are based on the so-called block-based hybrid video coding approach. The basic source-coding algorithm is a hybrid of inter-picture prediction to utilize temporal redundancy and transform coding of the prediction error signal to reduce spatial redundancy. Each picture of a video signal is partitioned into fixed-size macroblocks of $16 \times 16$ samples, which can be transmitted in one of several coding modes depending on the picture or slice coding type. Common to all standards is the definition of INTRA coded pictures or I-pictures. In I-pictures, all macroblocks are coded without referring to other pictures in the video sequence. Also common is the definition of predictive-coded pictures, so-called P-pictures and B-pictures, with the latter being extended conceptually in H.264/AVC coding. In predictive-coded pictures, typically one of a variety of INTER coding modes can be chosen to encode each macroblock.

In order to manage the large number of coding tools included in standards and the broad range of formats and bit rates supported, the concept of profiles and levels is typically employed to define a set of conformance points, each targeting a specific class of applications. These conformance points are designed to facilitate interoperability between various applications of the standard that have similar functional requirements. A profile defines a set of coding tools or algorithms that can be used in generating a compliant bitstream, whereas a level places constraints on certain key parameters of the bitstream, such as the picture resolution and bit rate.

Although MPEG-2, H.263, MPEG-4, and H.264/AVC define similar coding algorithms, they contain features and enhancements that make them differ. These differences mainly involve the formation of the prediction signal, the block sizes used for transform coding, and the entropy coding methods. In the following, the description of the various standards is limited to those features relevant to the comparisons described in this paper. 


\section{A. ISO/IEC Standard 13818-2/ITU-T Recommendation H.262: $M P E G-2$}

MPEG-2 forms the heart of broadcast-quality digital television for both standard-definition and high-definition television (SDTV and HDTV) [1], [12], [13]. MPEG-2 video (IS 13 818-2/ITU-T Recommendation H.262) was designed to encompass MPEG-1 [14] and to also provide high quality with interlaced video sources at bit rates in the range of 4-30 Mbit/s. Although usually thought of as an ISO standard, MPEG-2 video was developed as an official joint project of both the ISO/IEC JTC1 and ITU-T organizations, and was completed in late 1994.

MPEG-2 incorporates various features from H.261 and MPEG-1. It uses the basic coding structure that is still predominant today. For each macroblock, which consists of one $16 \times 16$ luminance block and two $8 \times 8$ chrominance blocks for 4:2:0 formatted video sequences, a syntax element indicating the macroblock coding mode (and signalling a quantizer change) is transmitted. While all macroblocks of I-pictures are coded in INTRA mode, macroblocks of P-pictures can be coded in INTRA, INTER-16 $\times 16$, or SKIP mode. For the SKIP mode, runs of consecutive skipped macroblocks are transmitted and the representation of the picture in the skipped region is represented using INTER prediction without adding any residual difference representation. In B-pictures, the prediction signal for the motion-compensated INTER-16 $\times 16$ mode can be formed by forward, backward, or bidirectionally interpolated prediction. The motion compensation is generally based on $16 \times 16$ blocks and utilizes half-pixel accurate motion vectors, with bilinear interpolation of half-pixel positions. The motion vectors are predicted from a single previously encoded motion vector in the same slice.

Texture coding is conducted using a DCT on blocks of $8 \times 8$ samples, and uniform scalar quantization (with the exception of the central dead-zone) is applied that can be adjusted using quantization values from 2 to 62 . Additionally, a perceptually weighted matrix based on the frequency of each transform coefficient (except the Intra DC coefficient) can be used. The entropy coding is performed using zig-zag scanning and two-dimensional run-level variable-length coding (VLC). There are two available VLC tables for transmitting the transform coefficient levels, of which one must be used for predictive-coded macroblocks and either can be used for INTRA macroblocks, as selected by the encoder on the picture level.

For the coding of interlaced video sources, MPEG-2 provides the concept of field pictures and field-coded macroblocks in frame pictures. The top and bottom field of an interlaced frame can be coded together as frame picture or as two separate field pictures. In addition to the macroblock coding modes described above, field-picture macroblocks can also be coded in INTER-16 $\times 8$ prediction mode, in which two different prediction signals are used, one for the upper and one for the lower half of a macroblock. For macroblocks in frame pictures, a similar coding mode is provided that uses different prediction signals for the top and bottom field lines of a macroblock. Macroblocks of both field and frame pictures can also be transmitted in dual prime mode. In this coding mode, the final prediction for each field is formed by averaging two prediction signals, of which one is obtained by referencing the field with the same parity and the other is obtained by referencing the field with the opposite parity as the current field. For coding of the residual data, MPEG-2 provides the possibility to use an alternative scanning pattern, which can be selected on picture level, and to choose between a frame- and field-based DCT coding of the prediction error signal.

The most widely implemented conformance point in the MPEG-2 standard is the Main profile at the Main Level (MP@ML). MPEG-2 MP@ML compliant encoders find application in DVD-video, digital cable television, terrestrial broadcast of standard definition television, and direct-broadcast satellite (DBS) systems. This conformance point supports coding of CCIR 601 content at bit rates up to $15 \mathrm{Mbit} / \mathrm{s}$ and permits use of B-pictures and interlaced prediction modes. In this work, an MPEG-2 encoder is included in the comparisons of video encoders for streaming and entertainment applications. The MPEG-2 bitstreams generated for our comparisons are compliant with the popular MP@ML conformance point with exception of the HDTV bitstreams, which are compliant with the MP@HL conformance point.

\section{B. ITU-T Recommendation H.263}

The first version of ITU-T Recommendation H.263 [2] defines a basic source-coding algorithm similar to that of MPEG-2, utilizing the INTER-16 × 16, INTRA, and SKIP coding modes. But H.263 Baseline contains significant changes that make it more efficient at lower bit rates including median motion vector prediction and three-dimensional run-level-last VLC with tables optimized for lower bit rates.

Moreover, version 1 of H.263 contains eight Annexes (Annexes A-G) including four Annexes permitting source coding options (Annexes D, E, F, and G) for improved compression performance. Annexes D and F are in frequent use today. Annex D specifies the option for motion vectors to point outside the reference picture and to have longer motion vectors than H.263 Baseline. Annex F specifies the use of overlapped block motion compensation and four motion vectors per macroblock with each motion vector assigned to an $8 \times 8$ subblock, i.e., the use of variable block sizes. Hence, an INTER- $8 \times 8$ coding mode is added to the set of possible macroblock modes.

H.263+ is the second version of H.263 [2], [15], where several optional features are added to H.263 as Annexes I through T. Annex J of H.263+ specifies a deblocking filter that is applied inside the motion prediction loop and is used together with the variable block-size feature of Annex F. H.263+ also adds some improvements in compression efficiency for the INTRA macroblock mode through prediction of intra-DCT transform coefficients from neighboring blocks and specialized quantization and VLC coding methods for intra coefficients. This advanced syntax is described in Annex I of the ITU-T Recommendation H.263+. Annex I provides significant rate-distortion improvements between 1 and $2 \mathrm{~dB}$ compared to the H.263 Baseline INTRA macroblock coding mode when utilizing the same amount of bits for both codecs [15]. Annex T of H.263+ removes some limitations of the Baseline syntax in terms of quantization and also improves chrominance fidelity by specifying a smaller 
step size for chrominance coefficients than for luminance. The remaining Annexes contain additional functionalities including specifications for custom and flexible video formats, scalability, and backward-compatible supplemental enhancement information.

A second set of extensions that adds three more optional modes to H.263 [2] was completed and approved late in the year 2000. This version is often referred to as H.263++. The data partitioned slice mode (Annex V) can provide enhanced resilience to bit-stream corruption, which typically occurs during transmission over wireless channels, by separating header and motion vector information from transform coefficients. Annex W specifies additional backward-compatible supplemental enhancement information including interlaced field indications, repeated picture headers, and the indication of the use of a specific fixed-point inverse DCT. Compression efficiency and robustness to packet loss can be improved by using the enhanced reference picture selection mode (Annex $\mathrm{U})$, which enables long-term memory motion compensation [22], [23]. In this mode, the spatial displacement vectors that indicate motion-compensated prediction blocks are extended by variable time delay, permitting the predictions to originate from reference pictures other than the most recently decoded reference picture. Motion-compensation performance is improved because of the larger number of possible predictions that are available by including more reference frames in the motion search. In Annex U, two modes are available for the buffering of reference pictures. The sliding-window mode-in which only the most recent reference pictures are stored-is the simplest and most commonly implemented mode. In the more flexible adaptive buffering mode, buffer management commands can be inserted into the bitstream as side information, permitting an encoder to specify how long each reference picture remains available for prediction, with a constraint on the total size of the picture buffer. The maximum number of reference pictures is typically 5 or 10 when conforming to one of H.263's normative profiles, which are discussed next.

The ITU-T has recently approved Annex X of H.263, which provides a normative definition of profiles, or preferred combinations of optional modes, and levels, which specify maximum values for several key parameters of an H.263 bitstream. Similar to their use in MPEG-2, each profile is designed to target a specific key application, or group of applications that require similar functionality. In this work, the rate-distortion capabilities of the Baseline profile and the Conversational High Compression $(\mathrm{CHC})$ profile are compared to other standards for use in videoconferencing applications. The Baseline profile supports only Baseline H.263 syntax (i.e., no optional modes) and exists to provide a profile designation to the minimal capability that all compliant decoders must support. The $\mathrm{CHC}$ profile includes most of the optional modes that provide enhanced coding efficiency without the added delay that is introduced by B-pictures and without any optional error resilience features. Hence, it is the best profile to demonstrate the optimal rate-distortion capabilities of the H.263 standard for use in interactive video applications. Additionally, the High-Latency (HL) profile of H.263, which adds support for B-pictures to the coding efficiency tools of the CHC profile, is included in the comparison of encoders for streaming applications, in which the added delay introduced by $\mathrm{B}$-pictures is acceptable.

\section{ISO/IEC Standard 14496-2: MPEG-4}

MPEG-4 Visual [3] standardizes efficient coding methods for many types of audiovisual data, including natural video content. For this purpose, MPEG-4 Visual uses the Baseline H.263 algorithm as a starting point so that all compliant MPEG-4 decoders must be able to decode any valid Baseline H.263 bitstream. However, MPEG-4 includes several additional features that can improve coding efficiency.

While spatial coding in MPEG- 4 uses the $8 \times 8$ DCT and scalar quantization, MPEG-4 supports two different scalar quantization methods that are referred to as MPEG-style and H.263-style. In the MPEG-style quantization, perceptually weighted matrices, similar to those used in MPEG-2 assign a specific quantizer to each coefficient in a block, whereas in the H.263 method, the same quantizer is used for all ac coefficients. Quantization of DC coefficients uses a special nonlinear scale that is a function of the quantization parameter. Quantized coefficients are scanned in a zig-zag pattern and assigned run-length codes, as in H.263. MPEG-4 also includes alternate scan patterns for horizontally and vertically predicted INTRA blocks and the use of a separate VLC table for INTRA coefficients. These techniques are similar to those defined in Annex I of H.263.

Motion compensation in MPEG- 4 is based on $16 \times 16$ blocks and supports variable block sizes, as in Annex F of H.263, so that one motion vector can be specified for each of the $8 \times 8$ subblocks of a macroblock, permitting the use of the INTER- $8 \times 8$ mode. Version 1 of MPEG-4 supports only motion compensation at half-pixel accuracy, with bilinear interpolation used to generate values at half-pixel positions. Version 2 of MPEG-4 additionally supports the use of quarter-pixel accurate motion compensation, with a windowed 8-tap sinc function used to generate half-pixel positions and bilinear interpolation for quarter-pixel positions. Motion vectors are permitted to point outside the reference picture and are encoded differentially after median prediction, according to H.263. MPEG-4 does not include a normative de-blocking filter inside the motion compensation loop, as in Annex J of H.263, but post filters may be applied to the reconstructed output at the decoder to improve visual quality.

The MPEG-4 Simple profile includes all features mentioned above, with the exception of the MPEG-style quantization method and quarter-pixel motion compensation. The Advanced Simple profile adds these two features, plus B-pictures, global motion compensation (GMC) and special tools for efficient coding of interlaced video. A video coder compliant with the Simple profile and the Advanced Simple profile will be used in our experiments.

\section{ITU-T Recommendation H.264/ISO/IEC Standard 14496-10 AVC: H.264/AVC}

H.264/AVC [4] is the latest joint project of the ITU-T VCEG and ISO/IEC MPEG. The H.264/AVC design covers a video coding layer (VCL) and a Network Adaptation Layer (NAL). 
Although the VCL design basically follows the design of prior video coding standards such as MPEG-2, H.263, and MPEG-4, it contains new features that enable it to achieve a significant improvement in compression efficiency in relation to prior coding standards. For details, please refer to [16]. Here, we will give a very brief description of the necessary parts of H.264/AVC in order to make the paper more self-contained.

In H.264/AVC, blocks of $4 \times 4$ samples are used for transform coding, and thus a macroblock consists of 16 luminance and 8 chrominance blocks. Similar to the I-, P-, and B-pictures defined for MPEG-2, H.263, and MPEG-4, the H.264/AVC syntax supports I-, P-, and B-slices. A macroblock can always be coded in one of several INTRA coding modes. There are two classes of INTRA coding modes, which are denoted as INTRA-16 $\times 16$ and INTRA- $4 \times 4$ in the following. In contrast to previous standards where only some of the DCT-coefficients can be predicted from neighboring INTRA-blocks, in H.264/AVC, prediction is always utilized in the spatial domain by referring to neighboring samples of already coded blocks. When using the INTRA- $4 \times 4$ mode, each $4 \times 4$ block of the luminance component utilizes one of nine prediction modes. The chosen modes are transmitted as side information. With the INTRA- $16 \times 16$ mode, a uniform prediction is performed for the whole luminance component of a macroblock. Four prediction modes are supported in the INTRA-16 $\times 16$ mode. For both classes of INTRA coding modes, the chrominance components are predicted using one of four possible prediction modes.

In addition to the INTRA modes, H.264/AVC provides various other motion-compensated coding modes for macroblocks in P-slices. Each motion-compensated mode corresponds to a specific partition of the macroblock into fixed size blocks used for motion description. Macroblock partitions with block sizes of $16 \times 16,16 \times 8,8 \times 16$, and $8 \times 8$ luminance samples are supported by the syntax corresponding to the INTER-16 $\times 16$, INTER-16 $\times 8$, INTER- $8 \times 16$, and INTER- $8 \times 8$ macroblock modes, respectively. In case the INTER- $8 \times 8$ macroblock mode is chosen, each of the $8 \times 8$ submacroblocks can be further partitioned into blocks of $8 \times 8,8 \times 4,4 \times 8$, or $4 \times 4$ luminance samples. H.264/AVC generally supports multi-frame motion-compensated prediction. That is, similar to Annex $\mathrm{U}$ of H.263, more than one prior coded picture can be used as reference for the motion compensation. In H.264/AVC, motion compensation is performed with quarter-pixel accurate motion vectors. Prediction values at half-pixel locations are obtained by applying a one-dimensional six-tap finite impulse response (FIR) filter in each direction requiring a half-sample offset (horizontal or vertical or both, depending on the value of the motion vector), and prediction values at quarter-pixel locations are generated by averaging samples at the integerand half-pixel positions. The motion vector components are differentially coded using either median or directional prediction from neighboring blocks.

In comparison to MPEG-2, H.263, and MPEG-4, the concept of B-slices is generalized in H.264/AVC. For details please refer to [17]. B-slices utilize two distinct reference picture lists, and four different types of INTER prediction are supported: list 0 , list 1 , bi-predictive, and direct prediction. While list 0 prediction indicates that the prediction signal is formed by motion compensation from a picture of the first reference picture list, a picture of the second reference picture list is used for building the prediction signal if list 1 prediction is used. In the bi-predictive mode, the prediction signal is formed by a weighted average of a motion-compensated list 0 and list 1 prediction signal. The direct prediction mode differs from the one used in H.263 and MPEG-4 in that no delta motion vector is transmitted. Furthermore, there are two methods for obtaining the prediction signal, referred to as temporal and spatial direct prediction, which can be selected by an encoder on the slice level. B-slices utilize a similar macroblock partitioning to P-slices. Besides the INTER-16 $\times 16$, INTER-16 $\times 8$, INTER- $8 \times 16$, INTER- $8 \times 8$ and the INTRA modes, a macroblock mode that utilizes direct prediction, the DIRECT mode, is provided. Additionally, for each $16 \times 16,16 \times 8,8 \times 16$, and $8 \times 8$ partition, the prediction method (list 0 , list 1 , bi-predictive) can be chosen separately. An $8 \times 8$ partition of a B-slice macroblock can also be coded in DIRECT- $8 \times 8$ mode. If no prediction error signal is transmitted for a DIRECT macroblock mode, it is also referred to as B-slice SKIP mode.

H.264/AVC is basically similar to prior coding standards in that it utilizes transform coding of the prediction error signal. However, in H.264/AVC the transformation is applied to $4 \times 4$ blocks and, instead of the DCT, H.264/AVC uses a separable integer transform with basically the same properties as a $4 \times 4$ DCT. Since the inverse transform is defined by exact integer operations, inverse-transform mismatches are avoided. An additional $2 \times 2$ transform is applied to the four DC coefficients of each chrominance component. If the INTRA $16 \times 16$-mode is in use, a similar operation extending the length of the transform basis functions is performed on the $4 \times 4 \mathrm{DC}$ coefficients of the luminance signal.

For the quantization of transform coefficients, H.264/AVC uses scalar quantization, but without an extra-wide dead-zone around zero as found in H.263 and MPEG-4. One of 52 quantizers is selected for each macroblock by the quantization parameter $Q$. The quantizers are arranged in a way that there is an increase of approximately $12.5 \%$ in quantization step size when incrementing $Q$ by one. The transform coefficient levels are scanned in a zig-zag fashion if the block is part of a macroblock coded in frame mode; for field-mode macroblocks, an alternative scanning pattern is used. The $2 \times 2 \mathrm{DC}$ coefficients of the chrominance components are scanned in raster-scan order. All syntax elements of a macroblock including the vectors of scanned transform coefficient levels are transmitted by entropy coding methods.

Two methods of entropy coding are supported by H.264/AVC. The default entropy coding method uses a single infinite-extend codeword set for all syntax elements except the residual data. The vectors of scanned transform coefficient levels are transmitted using a more sophisticated method called context-adaptive VLC (CAVLC). This scheme basically uses the concept of run-length coding as it is found in MPEG-2, H.263, and MPEG-4; however, VLC tables for various syntax elements are switched depending on the values of previously transmitted syntax elements. Since the VLC tables are well designed to match the corresponding conditional statistics, the entropy coding performance is improved in com- 
parison to schemes using a single VLC table. The efficiency of entropy coding can be improved further if the context-adaptive binary arithmetic coding (CABAC) is used. On the one hand, the usage of arithmetic coding allows the assignment of a noninteger number of bits to each symbol of an alphabet, which is extremely beneficial for symbol probabilities much greater than 0.5 . On the other hand, the usage of adaptive codes permits adaptation to nonstationary symbol statistics. Another important property of CABAC is its context modeling. The statistics of already coded syntax elements are used to estimate conditional probabilities of coding symbols. Inter-symbol redundancies are exploited by switching several estimated probability models according to already coded symbols in the neighborhood of the symbol to encode. For details about CABAC, please refer to [18].

For removing block-edge artifacts, the H.264/AVC design includes a de-blocking filter, which is applied inside the motion prediction loop. The strength of filtering is adaptively controlled by the values of several syntax elements.

Similar to MPEG-2, a frame of interlaced video can be coded as a single frame picture or two separate field pictures. Additionally, H.264/AVC supports a macroblock-adaptive switching between frame and field coding. Therefore, a pair of vertically adjacent macroblocks is considered as a coding unit, which can be either transmitted as two frame macroblocks or a top and a bottom field macroblock.

In H.264/AVC, three profiles are defined. The Baseline profile includes all described features except B-slices, CABAC, and the interlaced coding tools. Since the main target application area of the Baseline profile is the interactive transmission of video, it is used in the comparison of video encoders for videoconferencing applications. In the comparison for video streaming and entertainment applications, which allow a larger delay, the Main profile of H.264/AVC is used. The Main profile adds support for B-slices, the highly efficient CABAC entropy coding method, as well as the interlaced coding tools.

\section{VIDEO CODER CONTROL}

One key problem in video compression is the operational control of the source encoder. This problem is compounded because typical video sequences contain widely varying content and motion, necessitating the selection between different coding options with varying rate-distortion efficiency for different parts of the image. The task of coder control is to determine a set of coding parameters, and thereby the bitstream, such that a certain rate-distortion trade-off is achieved for a given decoder. This article focuses on coder control algorithms for the case of error-free transmission of the bitstream. For a discussion of the application of coder control algorithms in the case of error-prone transmission, please refer to [19]. A particular emphasis is on Lagrangian bit-allocation techniques, which have emerged to form the most widely accepted approach in recent standard development. The popularity of this approach is due to its effectiveness and simplicity. For completeness, we briefly review the Lagrangian optimization techniques and their application to video coding.

\section{A. Optimization Using Lagrangian Techniques}

Consider $K$ source samples that are collected in the $K$-tuple $\mathbf{S}=\left(\boldsymbol{S}_{1}, \ldots, \boldsymbol{S}_{\mathrm{K}}\right)$. A source sample $\boldsymbol{S}_{k}$ can be a scalar or vector. Each source sample $\boldsymbol{S}_{k}$ can be quantized using several possible coding options that are indicated by an index out of the set $\boldsymbol{O}_{k}=\left(O_{k 1}, \ldots, O_{k N_{k}}\right)$. Let $I_{k} \in \boldsymbol{O}_{k}$ be the selected index to code $S_{k}$. Then the coding options assigned to the elements in $\mathbf{S}$ are given by the components in the $K$-tuple $\mathbf{I}=\left(I_{1}, \ldots, I_{\mathrm{K}}\right)$. The problem of finding the combination of coding options that minimizes the distortion for the given sequence of source samples subject to a given rate constraint $R_{c}$ can be formulated as

$$
\begin{array}{rl}
\min _{\mathbf{I}} & D(\mathbf{S}, \mathbf{I}) \\
\text { subject to } & R(\mathbf{S}, \mathbf{I}) \leq R_{c} .
\end{array}
$$

Here, $D(\mathbf{S}, \mathbf{I})$ and $R(\mathbf{S}, \mathbf{I})$ represent the total distortion and rate, respectively, resulting from the quantization of $\mathbf{S}$ with a particular combination of coding options $\mathbf{I}$. In practice, rather than solving the constrained problem in (1), an unconstrained formulation is employed, that is

$$
\begin{aligned}
\mathbf{I}^{*} & =\underset{I}{\operatorname{argmin}} J(\mathbf{S}, \mathbf{I} \mid \lambda) \\
\text { with } J(\mathbf{S}, \mathbf{I} \mid \lambda) & =D(\mathbf{S I})+\lambda \cdot R(\mathbf{S I})
\end{aligned}
$$

and $\lambda \geq 0$ being the Lagrange parameter. This unconstrained solution to a discrete optimization problem was introduced by Everett [20]. The solution $\mathbf{I} *$ to (2) is optimal in the sense that if a rate constraint $R_{c}$ corresponds to $\lambda$, then the total distortion $D\left(\mathbf{S}, \mathbf{I}^{*}\right)$ is minimum for all combinations of coding options with bit rate less or equal to $R_{c}$.

We can assume additive distortion and rate measures, and let these two quantities be only dependent on the choice of the parameter corresponding to each sample. Then, a simplified Lagrangian cost function can be computed using

$$
J\left(S_{k}, \mathbf{I} \mid \lambda\right)=J\left(S_{k}, I_{k} \mid \lambda\right) .
$$

In this case, the optimization problem in (3) reduces to

$$
\min _{I} \sum_{k=1}^{K} J\left(S_{k}, \mathbf{I} \mid \lambda\right)=\sum_{k=1}^{K} \min _{I_{k}} J\left(S_{k}, I_{k} \mid \lambda\right)
$$

and can be easily solved by independently selecting the coding option for each $S_{k} \in \mathrm{S}$. For this particular scenario, the problem formulation is equivalent to the bit-allocation problem for an arbitrary set of quantizers, proposed by Shoham and Gersho [21].

\section{B. Lagrangian Optimization in Hybrid Video Coding}

The application of Lagrangian techniques to control a hybrid video coder is not straightforward because of temporal and spatial dependencies of the rate-distortion costs. Consider a blockbased hybrid video codec such as H.261, H.263, H.264/AVC or MPEG-1/2/4. Let the image sequence $s$ be partitioned into $K$ distinct blocks $\boldsymbol{A}_{k}$ and the associated pixels be given as $\boldsymbol{S}_{k}$. The options $\mathbf{O}_{k}$ to encode each block $\boldsymbol{S}_{k}$ are categorized into INTRA and INTER, i.e., predictive coding modes with associated parameters. The parameters are transform coefficients and quantizer value $Q$ for both modes plus one or more motion vectors for the INTER mode. The parameters for both modes 
are often predicted using transmitted parameters of preceding modes inside the image. Moreover, the INTER mode introduces a temporal dependency because reference is made to prior decoded pictures via motion compensated prediction. Hence, the optimization of a hybrid video encoder would require the minimization of the Lagrangian cost function in (2) for all blocks in the entire sequence. This minimization would have to proceed over the product space of the coding mode parameters. This product space is by far too large to be evaluated. Therefore, various publications elaborate on reductions of the product space and thus reducing complexity. For an overview, please refer to [24].

A simple and widely accepted method of INTER coding mode selection is to search for a motion vector that minimizes a Lagrangian cost criterion prior to residual coding. The bits and distortion of the following residual coding stage are either ignored or approximated. Then, given the motion vector(s), the parameters for the residual coding stage are encoded. The minimization of a Lagrangian cost function for motion estimation as given in (3) was first proposed by Sullivan and Baker [25].

Therefore, we split the problem of optimum bit allocation for INTER modes in a motion estimation and successive macroblock mode decision process between INTER or INTRA coding modes. The utilized macroblock mode decision is similar to [26] but without consideration of the dependencies of distortion and rate values on coding mode decisions made for past or future macroblocks. Hence, for each macroblock, the coding mode with associated parameters is optimized given the decisions made for prior coded blocks only. Consequently, the coding mode for each block is determined using the Lagrangian cost function in (3). Let the Lagrange parameter $\lambda_{\mathrm{MODE}}$ and the quantizer value $Q$ be given. The Lagrangian mode decision for a macroblock $\boldsymbol{S}_{k}$ proceeds by minimizing

$$
\begin{aligned}
& J_{\mathrm{MODE}}\left(\boldsymbol{S}_{k}, I_{k} \mid Q, \lambda_{\mathrm{MODE}}\right)= \\
& D_{\mathrm{REC}}\left(\boldsymbol{S}_{k}, I_{k} \mid Q\right)+\lambda_{\mathrm{MODE}} R_{\mathrm{REC}}\left(\boldsymbol{S}_{k}, I_{k} \mid Q\right)
\end{aligned}
$$

where the macroblock mode $I_{k}$ is varied over the sets of possible macroblock modes for the various standards. As an example, the following sets of macroblock modes can be used for P-pictures (or P-slices) when coding progressive-scanned video:

- MPEG-2: INTRA, SKIP, INTER-16 × 16;

- H.263/MPEG-4: INTRA, SKIP, INTER-16×16, INTER-8 $\times 8$;

- H.264/AVC: INTRA-4 $\times 4$, INTRA-16 $\times 16$, SKIP, INTER-16 $\times 16, \quad$ INTER-16 $\times 8, \quad$ INTER- $8 \times 16$, INTER- $8 \times 8$.

Please note that although sometimes named identically here, the various modes are different between the above various standards.

H.264/AVC additionally provides the following set of submacroblock types for each $8 \times 8$ submacroblock of a P-slice macroblock that is coded in INTER- $8 \times 8$ mode: INTER- $8 \times 8$, INTER- $8 \times 4$, INTER- $4 \times 8$, and INTER- $4 \times 4$.

The distortion $D_{\mathrm{REC}}\left(\boldsymbol{S}_{k}, I_{k} \mid Q\right)$ and rate $R_{\mathrm{REC}}\left(S_{k}, I_{k} \mid Q\right)$ for the various modes are computed as follows: For the INTRA modes, the corresponding $8 \times 8$ (MPEG-2, H.263/MPEG-4) or
$4 \times 4$ (H.264/AVC) blocks of the macroblock $\boldsymbol{S}_{k}$ are processed by transformation and subsequent quantization. The distortion $D_{\mathrm{REC}}\left(\boldsymbol{S}_{k}, \mathrm{INTRA} \mid Q\right)$ is measured as the sum of the squared differences (SSD) between the reconstructed $\left(s^{\prime}\right)$ and the original $(s)$ macroblock pixels

$$
\mathrm{SSD}=\sum_{(x, y) \in A}\left|s[x, y, t]-s^{\prime}[x, y, t]\right|^{2}
$$

where $\boldsymbol{A}$ is the subject macroblock. The rate $R_{\mathrm{REC}}\left(\boldsymbol{S}_{k}, \mathrm{INTRA} \mid Q\right)$ is the rate that results after entropy coding.

For the SKIP mode, the distortion $D_{\operatorname{REC}}\left(\boldsymbol{S}_{k}, \operatorname{SKIP} \mid Q\right)$ and rate $R_{\mathrm{REC}}\left(\boldsymbol{S}_{k}, \mathrm{SKIP} \mid Q\right)$ do not depend on the current quantizer value. The distortion is determined by the SSD between the current picture and the value of the inferred INTER prediction, and the rate is given as one bit per macroblock for H.263 and MPEG-4, and approximately one bit per macroblock for MPEG-2 and H.264/AVC.

The computation of the Lagrangian costs for the INTER modes is much more demanding than for the INTRA and SKIP modes. This is because of the block motion estimation step. The size of the blocks $S_{i}$ within a macroblock is $A \times B$ pixels for the INTER- $A \times B$ mode. Given the Lagrange parameter $\lambda_{\text {MOTION }}$ and the decoded reference picture $s^{\prime}$, rate-constrained motion estimation for a block $S_{i}$ is performed by minimizing the Lagrangian cost function

$$
\begin{aligned}
\boldsymbol{m}_{i}=\arg \min _{m \in M}\left\{D_{D F D}\left(\boldsymbol{S}_{i}, \boldsymbol{m}\right)\right. \\
\left.+\lambda_{\text {MOTION }} R_{\mathrm{MOTION}}\left(\boldsymbol{S}_{i}, \mathbf{m}\right)\right\}
\end{aligned}
$$

where $M$ is the set of possible coding modes and with the distortion term being given by

$$
\begin{aligned}
& D_{D F D}\left(\boldsymbol{S}_{i}, \boldsymbol{m}\right) \\
& \quad=\sum_{(x, y) \in A_{i}}\left|s[x, y, t]-s^{\prime}\left[x-m_{x}, y-m_{y}, t-m_{t}\right]\right|^{p}
\end{aligned}
$$

with $p=1$ for the SAD and $p=2$ for the SSD. $R_{\text {MOTION }}\left(S_{i}, \boldsymbol{m}\right)$ is the number of bits to transmit all components of the motion vector $\left(m_{x}, m_{y}\right)$, and, in case multiple reference frames are used, $m_{t}$. The search range $M$ is \pm 32 integer pixel positions horizontally and vertically and either 1 or more prior decoded pictures are referenced. Depending on the use of SSD or SAD, the Lagrange parameter $\lambda_{\text {MOTION }}$ has to be adjusted.

The motion search that minimizes (7) proceeds first over integer-pixel locations. Then, the best of those integer-pixel motion vectors is tested whether one of the surrounding half-pixel positions provides a cost reduction in (7). This procedure of determination of a subpixel position is called half-pixel refinement. In the case quarter-pixel motion accuracy is used, the previously determined half-pixel location is used as the center for the corresponding subpixel refinement step, respectively. The subpixel refinement yields the resulting motion vector $\boldsymbol{m}_{i}$. The resulting prediction error signal $u\left[x, y, t, \boldsymbol{m}_{i}\right]$ is processed by transformation and subsequent quantization, as in the INTRA mode case. The distortion $D_{\mathrm{REC}}$ is also measured as the SSD 
between the reconstructed and the original macroblock pixels. The rate $R_{\mathrm{REC}}$ is given as the sum of the bits for the mode information, the motion vectors as well as the transform coefficients.

A final remark should be made regarding the choice of the Lagrange parameters $\lambda_{\text {MODE }}$ and $\lambda_{\text {MOTION }}$. In [24], [27] it was shown via experimental results that the following relationship is efficient for H.263/MPEG-4:

$$
\lambda_{\mathrm{MODE}}=0.85 \cdot Q_{H .263}^{2}
$$

and for SAD in (7)

$$
\lambda_{\mathrm{MOTION}}=\sqrt{\lambda_{\mathrm{MODE}}} .
$$

Correspondingly, for SSD in (7), we would use

$$
\lambda_{\mathrm{MOTION}}=\lambda_{\mathrm{MODE}} \cdot
$$

The experiment that lead to the relationship in (8) has also been conducted for H.264/AVC providing the following equation:

$$
\lambda_{\mathrm{MODE}}=0.85 \cdot 2^{\left(Q_{H .264}-12\right) / 3}
$$

for $\lambda_{\mathrm{MODE}}$, and (10) and (11) for $\lambda_{\mathrm{MOTION}}$.

Thus, rate control in those codecs is conducted via controlling for instance the quantization parameter and adjusting the Lagrange parameters accordingly using (9)-(12).

\section{COMPARISON}

We performed three separate experiments, each targeting a particular application area. The first experiment evaluates performance for video streaming while the second experiment targets videoconferencing. The coding features used in these two applications differ primarily in that the low delay constraints that are imposed in the videoconferencing experiment are relaxed in the video streaming case. Additionally, appropriate content is selected to represent each application space. The third experiment addresses entertainment-quality applications. In this experiment, the coding features are similar to those used in the video streaming case, but high-resolution video sources are used.

\section{A. Video Streaming Applications}

Table I shows results for a set of test sequences and test conditions selected to represent a video streaming application. We have measured performance using Lagrangian RD-optimized encoders for this set of test conditions when encoding in conformance to four different standards/profiles: MPEG-2 MP, H.263 HLP, MPEG-4 ASP, and H.264/AVC MP (using the latest reference software JM-61e). Details about the input sequences used in the tests are listed in Appendix B. All coders used only one I-picture at the beginning of a sequence, and two B-pictures have been inserted between each two successive P-pictures. Full search motion estimation with a range of \pm 32 integer pixels was used by all encoders along with the Lagrangian Coder Control described in Section III.

The MPEG-2 Visual encoder generated bitstreams that are compliant with the popular ML@MP conformance point and the H.263 encoder used the HLP features. For MPEG-4
Visual, the ASP was used with quarter-sample accurate motion compensation and global motion compensation enabled. Additionally, the recommended de-blocking/de-ringing filter was applied as a post-processing operation. For the H.264/AVC JM-61e coder, the Main profile was used with CABAC as entropy coding method. We have generally used five reference frames for both H.263 and H.264/AVC. The usage of B-pictures in the H.264/AVC encoder was restricted in a way that B-pictures are not used as reference pictures, and that all preceding reference pictures (in decoding order) are inserted in reference picture list 0 , while only the future reference picture is placed in reference picture list 1 . That is, this restricted B-picture concept for H.264/AVC used in the comparison is very similar to that of MPEG-2, H.263, and MPEG-4. To achieve the target bit rate, a fixed quantization paramater setting was selected that resulted in the bit rate shown (with a change in the quantization parameter setting at one point during the sequence to fine-tune the target rate).

The target bit rates were always hit with a difference of approximately $2 \%$, except in two cases for MPEG-2 MP that are marked with "*”, where using the maximum step size did not result in a sufficiently low bit rate. The quantization parameter for B-pictures was set in such a way that the corresponding quantization step size was approximately $20 \%$ larger than that for P-pictures for all codecs. Table I shows that with the H.264/AVC compliant encoder, performance gains of 1-3 dB are achieved in comparison with the MPEG-4 coder, 1-5 dB are achieved in comparison with H.263, and 3-6 dB are achieved in comparison with MPEG-2.

In the left column of Fig. 1, rate-distortion curves for the four codecs are plotted for selected sequences. The test points shown in Table I are marked inside the plots by white circles. For all sequences, H.264/AVC significantly outperforms the other codecs. In the right column of Fig. 1 the bit-rate savings relative to the worst tested video coding standard, MPEG-2, is plotted against the PSNR of the luminance component for H.263 HLP, MPEG-4 ASP, and H.264/AVC MP.

Table II presents the average bit-rate savings provided by each encoder relative to all other tested encoders over the entire set of sequences and bit rates. It can be seen that H.264/AVC Coding significantly outperforms all other standards. On the most complex sequence of the test set, Mobile \& Calendar (CIF, $30 \mathrm{~Hz}$ ), average bit-savings of more than $70 \%$ relative to MPEG-2 are realized. Bit-rate savings are as low as 50\% on the Flower Garden sequence in CIF resolution $(15 \mathrm{~Hz})$, with an average of $63 \%$ over the entire test set. H.264/AVC Main profile provides more than $35 \%$ bit-rate savings relative to its two nearest competitors, MPEG-4 Advanced Simple and H.263 CHC. Note that H.264/AVC includes all of the main technical features used in these other encoder configurations, plus several additional features. The highly flexible motion model, the loop filtering, and the very efficient context-based arithmetic coding scheme are the three primary factors that enable the superior rate-distortion performance of H.264/AVC Main profile.

\section{B. Videoconferencing Applications}

This experiment evaluates coding performance for interactive video applications, such as videoconferencing, in which 
TABLE I

FiXed Bit-RATE REsUlts For VIDEO StREAMING APPlications

\begin{tabular}{|c|c|c|c|c|c|c|c|c|c|c|c|c|c|c|c|c|}
\hline \multirow[b]{2}{*}{ Sequence } & \multicolumn{4}{|c|}{ MPEG-2 } & \multicolumn{4}{|c|}{ H.263 HLP } & \multicolumn{4}{|c|}{ MPEG-4 ASP } & \multicolumn{4}{|c|}{ H.264/AVC MP } \\
\hline & Rate & PSNR-Y & PSNR-U & PSNR-V & Rate & PSNR-Y & PSNR-U & PSNR-V & Rate & PSNR-Y & PSNR-U & PSNR-V & Rate & PSNR-Y & PSNR-U & PSNR-V \\
\hline \multicolumn{17}{|c|}{ A: QCIF, $10 \mathrm{~Hz}, 32 \mathrm{kbit} / \mathrm{s}$} \\
\hline Foreman & 32.12 & 27.81 & 35.14 & 34.96 & 32.18 & 29.90 & 37.73 & 37.70 & 31.92 & 30.09 & 37.33 & 37.33 & 31.49 & 32.40 & 38.68 & 38.98 \\
\hline Container & 32.22 & 32.71 & 39.75 & 39.04 & 31.97 & 35.96 & 41.38 & 41.12 & 31.82 & 36.42 & 42.46 & 42.23 & 31.89 & 38.57 & 43.00 & 42.98 \\
\hline News & 32.44 & 29.97 & 35.07 & 36.75 & 32.50 & 34.06 & 38.68 & 39.31 & 32.24 & 33.30 & 37.50 & 38.58 & 31.96 & 35.75 & 39.45 & 40.05 \\
\hline Tempete & $36.91^{*}$ & 24.83 & 29.38 & 32.04 & 32.24 & 26.62 & 32.50 & 34.74 & 31.68 & 27.87 & 31.61 & 34.21 & 31.83 & 29.62 & 33.58 & 36.02 \\
\hline \multicolumn{17}{|c|}{ B: QCIF, $15 \mathrm{~Hz}, 64 \mathrm{kbit} / \mathrm{s}$} \\
\hline Foreman & 63.45 & 30.36 & 37.07 & 37.28 & 65.14 & 32.38 & 38.65 & 38.93 & 64.38 & 32.81 & 38.73 & 39.15 & 63.42 & 35.21 & 40.00 & 40.67 \\
\hline Container & 63.95 & 34.34 & 40.95 & 40.40 & 63.97 & 38.26 & 43.32 & 43.22 & 63.87 & 38.47 & 44.21 & 43.87 & 63.67 & 40.67 & 44.80 & 44.92 \\
\hline News & 63.45 & 32.61 & 37.33 & 38.55 & 63.80 & 36.25 & 39.79 & 40.43 & 64.00 & 35.78 & 39.37 & 40.67 & 63.98 & 38.80 & 41.71 & 42.27 \\
\hline Tempete & 65.21 & 26.36 & 30.65 & 33.14 & 64.39 & 28.39 & 33.34 & 35.57 & 64.13 & 29.39 & 32.59 & 35.14 & 63.43 & 31.78 & 34.65 & 36.89 \\
\hline \multicolumn{17}{|c|}{ C: CIF, 15Hz, $128 \mathrm{kbit} / \mathrm{s}$} \\
\hline Foreman & 130.37 & 28.94 & 35.78 & 36.30 & 128.40 & 30.91 & 38.35 & 39.26 & 127.83 & 31.30 & 38.16 & 38.99 & 128.70 & 33.66 & 39.49 & 40.87 \\
\hline Container & 127.90 & 32.63 & 39.94 & 39.95 & 129.02 & 34.99 & 42.00 & 41.84 & 128.62 & 35.28 & 42.16 & 41.91 & 128.67 & 36.74 & 42.40 & 42.40 \\
\hline News & 129.84 & 32.73 & 37.92 & 38.98 & 129.02 & 36.68 & 40.82 & 41.47 & 126.97 & 35.71 & 39.20 & 40.58 & 128.25 & 38.21 & 41.21 & 42.09 \\
\hline Tempete & $165.75^{*}$ & 25.60 & 30.67 & 33.33 & 129.07 & 26.47 & 33.42 & 35.66 & 129.11 & 27.51 & 32.03 & 34.78 & 126.34 & 29.16 & 34.41 & 36.71 \\
\hline \multicolumn{17}{|c|}{ D: CIF, $15 \mathrm{~Hz}$, } \\
\hline Bus & 260.78 & 25.96 & 35.78 & 36.25 & 258.76 & 26.97 & 37.60 & 38.87 & 256.15 & 28.31 & 37.57 & 39.15 & 256.14 & 29.86 & 38.44 & 39.96 \\
\hline Mobile & 256.01 & 24.59 & 29.96 & 30.17 & 259.20 & 25.66 & 31.97 & 32.40 & 258.88 & 27.07 & 32.24 & 32.63 & 254.87 & 29.73 & 34.26 & 34.69 \\
\hline Flower & 261.67 & 23.93 & 28.82 & 32.37 & 257.85 & 24.89 & 31.58 & 33.56 & 255.97 & 26.07 & 30.89 & 33.90 & 257.89 & 28.08 & 33.02 & 35.08 \\
\hline Tempete & 257.65 & 27.68 & 32.45 & 34.82 & 259.28 & 29.06 & 34.54 & 36.75 & 256.58 & 29.86 & 34.09 & 36.60 & 254.37 & 31.74 & 35.83 & 37.98 \\
\hline \multicolumn{17}{|c|}{$\mathrm{E}: \mathrm{CIF}, 30 \mathrm{~Hz}, 512 \mathrm{kbit} / \mathrm{s}$} \\
\hline Bus & 506.29 & 27.35 & 36.43 & 37.62 & 511.98 & 28.77 & 38.16 & 39.41 & 511.88 & 29.75 & 38.28 & 39.89 & 511.85 & 31.89 & 39.29 & 40.85 \\
\hline Mobile & 506.26 & 25.31 & 30.26 & 30.47 & 513.05 & 26.74 & 32.40 & 32.85 & 505.03 & 28.36 & 33.12 & 33.54 & 512.58 & 31.27 & 35.18 & 35.65 \\
\hline Flower & 518.64 & 25.71 & 30.25 & 33.08 & 517.90 & 26.35 & 31.99 & 34.14 & 511.76 & 27.96 & 32.16 & 34.79 & 514.59 & 30.16 & 33.95 & 35.67 \\
\hline Tempete & 521.40 & 28.43 & 32.91 & 35.14 & 513.73 & 29.45 & 34.94 & 37.11 & 510.55 & 30.84 & 34.74 & 37.18 & 515.49 & 32.79 & 36.36 & 38.38 \\
\hline \multicolumn{17}{|c|}{ F: CIF, $30 \mathrm{~Hz}, 1024 \mathrm{kbit} / \mathrm{s}$} \\
\hline Bus & 1022.54 & 30.72 & 38.70 & 40.12 & 1025.80 & 31.91 & 39.55 & 41.21 & 1022.54 & 32.82 & 39.94 & 41.60 & 1025.51 & 35.24 & 40.77 & 42.59 \\
\hline Mobile & 1029.58 & 28.16 & 33.00 & 33.27 & 1024.27 & 29.82 & 34.43 & 34.83 & 1029.18 & 31.37 & 35.29 & 35.74 & 1026.00 & 34.64 & 37.27 & 37.74 \\
\hline Flower & 1034.33 & 28.66 & 32.92 & 35.10 & 1033.05 & 29.77 & 33.77 & 35.27 & 1024.30 & 31.20 & 34.58 & 36.61 & 1020.08 & 33.67 & 36.23 & 37.32 \\
\hline Tempete & 1029.56 & 31.30 & 35.17 & 37.13 & 1022.81 & 32.55 & 36.53 & 38.50 & 1025.77 & 33.34 & 36.51 & 38.69 & 1020.06 & 35.54 & 37.90 & 39.68 \\
\hline
\end{tabular}

The achieved bit rates are given in kilobits per second, and the PSNR values for the luminance and the two chrominance components are given in decibels.

The symbol "*” marks two cases in which the target bit rate was significantly exceeded in the experiment despite use of the maximum quantization step size.

a small delay and real-time encoding capability are the key requirements. Such applications generally support low to medium bit rates and picture resolutions, with QCIF resolution at $10-128 \mathrm{kbit} / \mathrm{s}$ and CIF resolution at $128-512 \mathrm{kbit} / \mathrm{s}$ being the most common. The set of input sequences for this comparison consists of four QCIF (10 and $15 \mathrm{~Hz}$ ) and four CIF (15 and 30 $\mathrm{Hz}$ ) sequences. Refer to Appendix B for details about these sequences. Encoders included in this comparison are compliant with the following standards/profiles: the H.263 Baseline and CHC profiles, the MPEG-4 Simple profile, and the H.264/AVC Baseline profile.

H.264/AVC Main profile was not included in the experiment results shown in Fig. 2, although it ideally should have been, since it has compression performance capability that significantly exceeds that of the H.264/AVC Baseline profile and since the design of its $\mathrm{B}$ slices can be used in a way that avoids the extra delay previously associated with bi-predictive coding. This omission was for expediency, as significant effort would have been needed to make our encoding software capable of low-delay operation with a good use of this capability. The additional advantage expected from use of the H.264/AVC Main profile is in the range of $10 \%-20 \%$ in bit-rate savings, resulting primarily from the low-delay use of bi-prediction (i.e., bi-prediction using only temporally preceding reference pictures) and from the improved entropy coding performance of CABAC.

In all bitstreams, only the first picture was intra coded, with all of the subsequent pictures being temporally predicted (P-pictures). Both the H.263 CHC and H.264/AVC Baseline encoders used five reference pictures for long-term prediction. (This is the maximum number allowed for CIF sequences in Level 40 of H.263's normative profile and level definitions). A motion search range of \pm 32 integer pixels was employed by all encoders with the exception of H.263 Baseline, which is constrained by its syntax to a maximum range of \pm 16 integer pixels.

Since profiles are used to indicate decoder support for a set of optional modes, an encoder that is compliant with a particular profile is permitted-but not required - to use any of the 

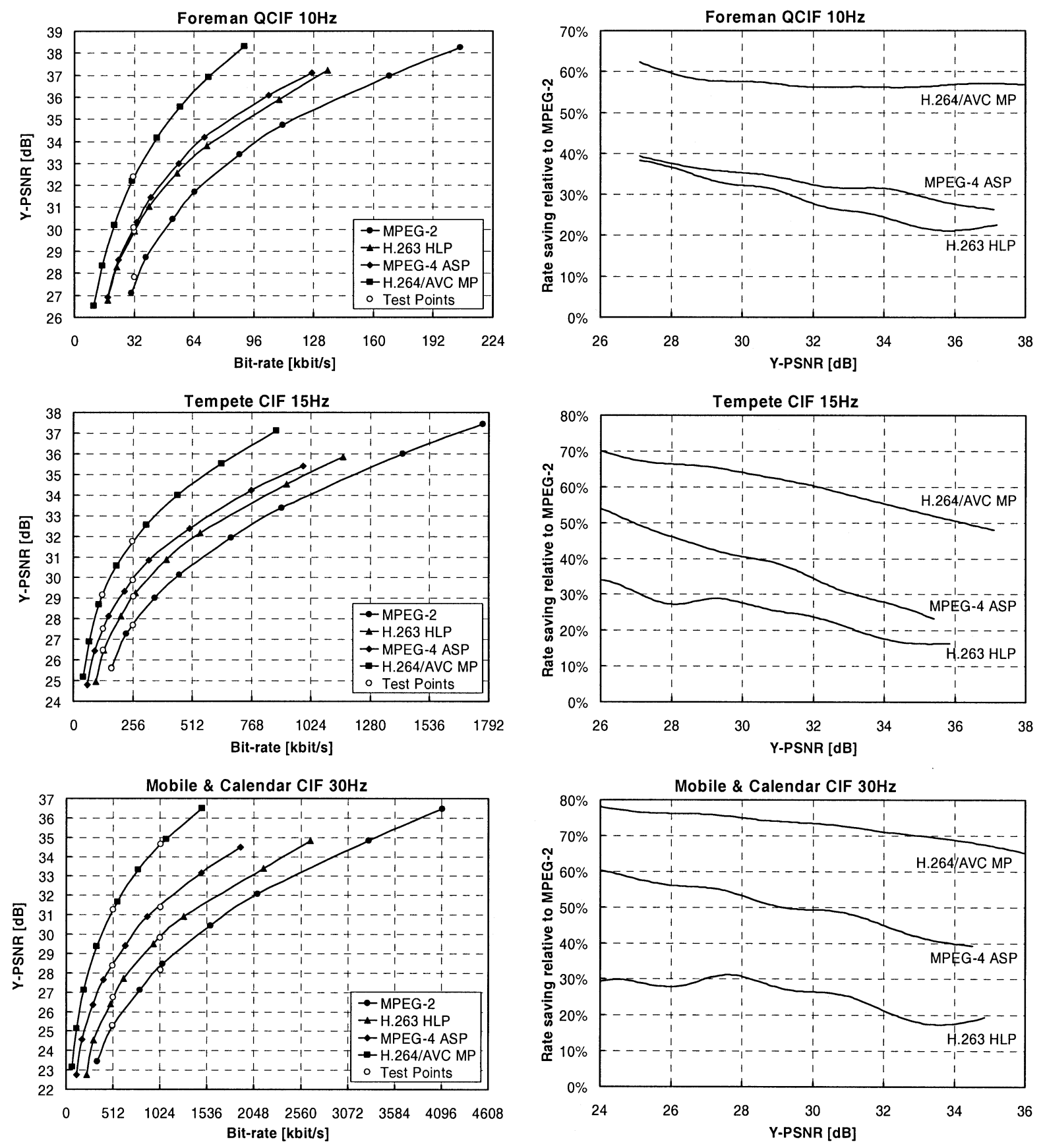

Fig. 1. Selected rate-distortion curves and bit-rate savings plots for video streaming applications.

TABLE II

Average Bit-Rate SAVINGS FOR Video Streaming ApPLicAtions

\begin{tabular}{c|c|c|c}
\hline & \multicolumn{3}{|c}{ Average bit-rate savings relative to: } \\
\hline Coder & MPEG-4 ASP & H.263 HLP & MPEG-2 \\
\hline H.264/AVC MP & $37.44 \%$ & $47.58 \%$ & $63.57 \%$ \\
\hline MPEG-4 ASP & - & $16.65 \%$ & $42.95 \%$ \\
\hline H.263 HLP & - & - & $30.61 \%$ \\
\hline
\end{tabular}

optional modes supported in a that profile. With this in mind, encoders were configured by only including the optional modes from each profile that would produce the best possible rate-distortion performance, while satisfying the low delay and complexity requirements of interactive video applications.
As in the first experiment, we present both rate-distortion curves for luminance component, as well as plots of bit-rate savings relative to the poorest performing encoder. As should be expected, it is the H.263 Baseline encoder that provides the worst rate-distortion performance, and therefore it serves as the common basis for comparison. Fig. 2 shows the rate-distortion plots as well as the bit-rate savings plots for three selected test sequences. The average bit-rate savings results over the entire test set are given in Table III. In addition to the selected rate-distortion and bit-rate savings plots of Fig. 2, results for fixed target bit rates between $24 \mathrm{kbit} / \mathrm{s}$ for $10-\mathrm{Hz}$ QCIF sequences and 256 kbits/s for 30-Hz CIF sequences are shown in Table IV.

It is immediately clear from these results that the next-generation H.264/AVC standard outperforms all of the other 

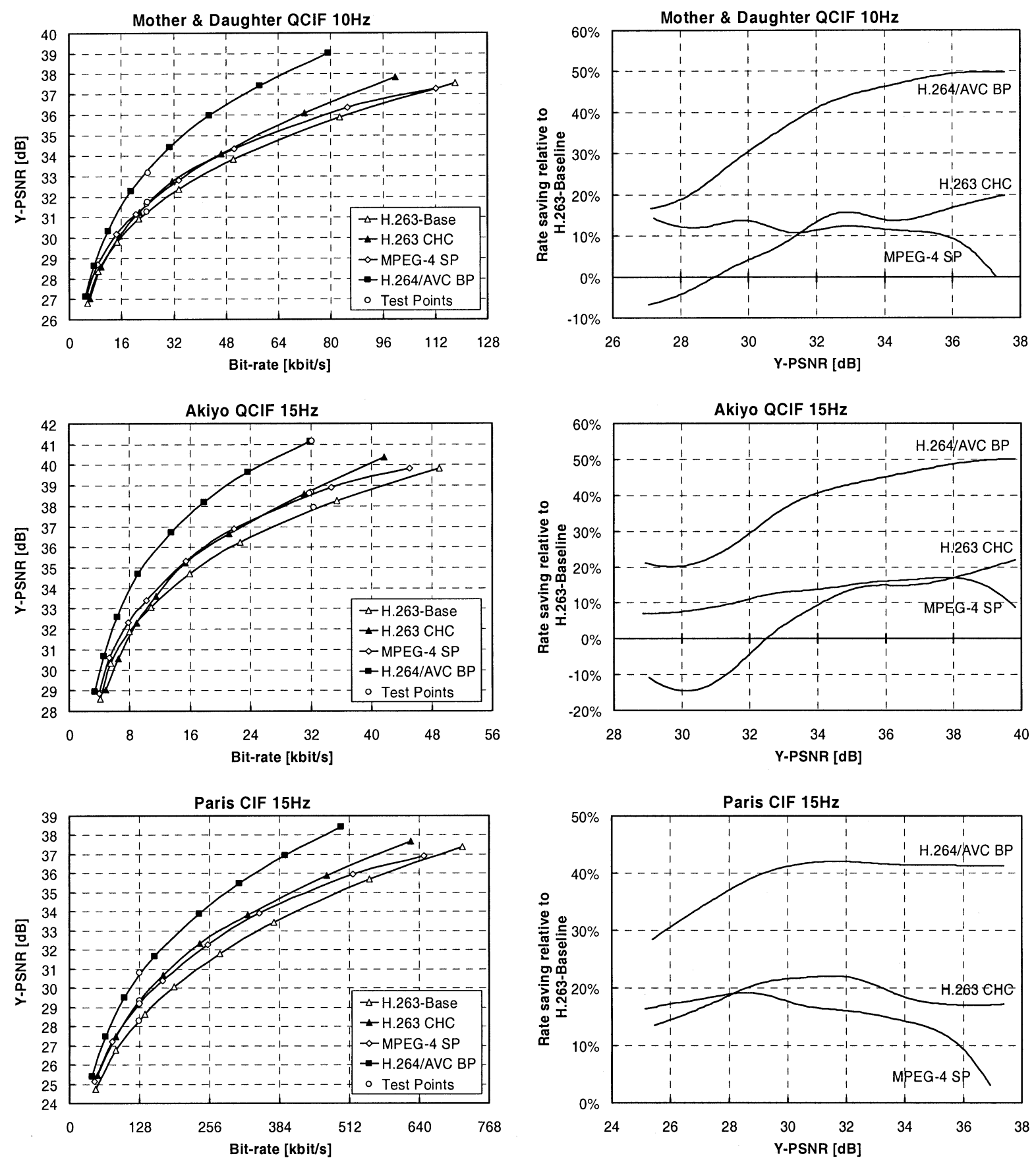

Fig. 2. Selected rate-distortion curves and bit-rate savings plot for videoconferencing applications (neglecting low-delay H.264/AVC MP).

TABLE III

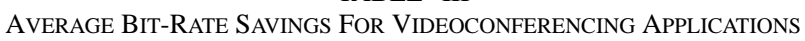

\begin{tabular}{c|c|c|c}
\hline & \multicolumn{3}{|c}{ Average bit-rate savings relative to: } \\
\hline Coder & H.263 CHC & MPEG-4 SP & H.263 Base \\
\hline H.264/AVC BP & $27.69 \%$ & $29.37 \%$ & $40.59 \%$ \\
\hline H.263 CHC & - & $2.04 \%$ & $17.63 \%$ \\
\hline MPEG-4 SP & - & - & $15.69 \%$ \\
\hline
\end{tabular}

(Neglecting low-delay H.264/AVC MP)

standards by a substantial margin. Bit-rate savings of more than $40 \%$ relative to $\mathrm{H} .263$ Baseline are realized. Relative to H.263 CHC, H.264/AVC provides more than $25 \%$ bit-rate savings. These reported bit-rate savings are lower than the bit-rate savings measured in the first experiment for video streaming applications. This is mainly related to the fact that we have chosen typical videophone/videoconferencing sequences for the second experiment. These sequences are generally characterized by low or medium motion as well as low spatial detail. However, for H.264/AVC, the largest improvements of coding efficiency are obtained for complex sequences such as Mobile \& Calendar. Furthermore, the H.264/AVC MP results for video streaming applications benefit from the usage of the highly efficient context-based arithmetic coding scheme that is not included in the Baseline profile of H.264/AVC.

By examining the relative rate-distortion performance of various standards and profiles included in this experiment, further insight into the gains in coding efficiency provided by 
TABLE IV

FIXED BIT-RATE RESULTS FOR VIDEOCONFERENCING APPLICATIONS

\begin{tabular}{|c|c|c|c|c|c|c|c|c|c|c|c|c|c|c|c|c|}
\hline \multirow[b]{2}{*}{ Sequence } & \multicolumn{4}{|c|}{ H.263 Baseline } & \multicolumn{4}{|c|}{ H.263 CHC } & \multicolumn{4}{|c|}{ MPEG-4 SP } & \multicolumn{4}{|c|}{ H.264/AVC Baseline } \\
\hline & Rate & PSNR-Y & PSNR-U & PSNR-V & Rate & PSNR-Y & PSNR-U & PSNR-V & Rate & PSNR-Y & PSNR-U & PSNR-V & Rate & PSNR-Y & PSNR-U & PSNR-V \\
\hline \multicolumn{17}{|c|}{ A: QCIF, $10 \mathrm{~Hz}, 24 \mathrm{kbit} / \mathrm{s}$} \\
\hline Akiyo & 24.14 & 37.34 & 39.73 & 41.31 & 24.06 & 38.54 & 41.89 & 42.93 & 24.19 & 38.01 & 40.24 & 41.95 & 24.00 & 40.68 & 42.90 & 43.58 \\
\hline Foreman & 24.21 & 27.73 & 35.39 & 34.95 & 24.25 & 28.52 & 37.39 & 37.37 & 24.09 & 29.10 & 36.27 & 35.95 & 23.87 & 30.08 & 37.45 & 37.58 \\
\hline Mother \& Daughter & 23.78 & 31.27 & 36.49 & 36.32 & 23.82 & 31.68 & 37.80 & 37.65 & 23.97 & 31.75 & 36.62 & 36.37 & 24.08 & 33.19 & 37.96 & 37.71 \\
\hline Silent & 24.08 & 31.12 & 35.44 & 36.93 & 23.90 & 32.31 & 37.28 & 38.83 & 24.14 & 31.68 & 35.51 & 37.02 & 24.09 & 32.42 & 36.34 & 38.07 \\
\hline \multicolumn{17}{|c|}{ B: QCIF, $15 \mathrm{~Hz}, 32 \mathrm{kbit} / \mathrm{s}$} \\
\hline Akiyo & 32.31 & 37.93 & 40.53 & 41.87 & 32.05 & 38.68 & 41.97 & 42.98 & 31.76 & 38.62 & 41.12 & 42.60 & 32.07 & 41.15 & 43.22 & 43.95 \\
\hline Foreman & 31.78 & 28.17 & 35.38 & 35.01 & 32.10 & 28.66 & 37.39 & 37.34 & 32.13 & 29.35 & 36.19 & 36.13 & 32.37 & 30.51 & 37.58 & 37.60 \\
\hline Mother \& Daughter & 31.77 & 31.56 & 36.62 & 36.49 & 31.74 & 31.87 & 37.81 & 37.61 & 32.27 & 31.96 & 36.73 & 36.70 & 32.14 & 33.66 & 37.99 & 37.81 \\
\hline Silent & 31.79 & 31.21 & 35.46 & 36.90 & 31.88 & 32.58 & 37.58 & 38.89 & 31.97 & 31.95 & 35.74 & 37.39 & 32.18 & 32.47 & 36.45 & 38.04 \\
\hline \multicolumn{17}{|c|}{ C: CIF, $15 \mathrm{~Hz}, 128 \mathrm{kbit} / \mathrm{s}$} \\
\hline Carphone & 129.71 & 31.53 & 35.94 & 37.03 & 127.64 & 32.32 & 38.02 & 39.24 & 127.82 & 32.50 & 36.62 & 37.73 & 125.64 & 33.50 & 37.75 & 39.23 \\
\hline Foreman & 128.32 & 29.92 & 36.40 & 37.00 & 127.97 & 30.76 & 38.50 & 39.39 & 128.65 & 31.52 & 37.71 & 38.45 & 127.24 & 32.96 & 38.77 & 40.06 \\
\hline Paris & 127.38 & 28.30 & 33.30 & 33.84 & 128.29 & 29.34 & 35.56 & 36.32 & 127.95 & 29.18 & 33.59 & 34.25 & 128.52 & 30.81 & 35.80 & 36.18 \\
\hline Sean & 129.74 & 36.64 & 40.56 & 41.07 & 128.47 & 37.91 & 41.71 & 42.29 & 127.37 & 36.75 & 40.50 & 41.31 & 129.89 & 39.46 & 42.22 & 43.05 \\
\hline \multicolumn{17}{|c|}{ D: CIF, $30 \mathrm{~Hz}, 256 \mathrm{kbit} / \mathrm{s}$} \\
\hline Carphone & 258.89 & 32.47 & 36.35 & 37.54 & 256.20 & 33.31 & 38.20 & 39.62 & 256.71 & 33.34 & 36.99 & 38.20 & 257.42 & 34.39 & 37.79 & 39.21 \\
\hline Foreman & 254.66 & 31.60 & 37.23 & 37.86 & 256.49 & 32.06 & 38.96 & 40.05 & 258.48 & 32.39 & 38.08 & 39.03 & 253.62 & 34.27 & 39.59 & 40.85 \\
\hline Paris & 257.05 & 29.55 & 34.08 & 34.70 & 258.19 & 30.56 & 36.19 & 36.65 & 254.91 & 30.34 & 34.44 & 34.95 & 256.43 & 32.24 & 36.67 & 36.93 \\
\hline Sean & 254.91 & 37.94 & 41.42 & 42.06 & 258.52 & 39.53 & 43.03 & 43.65 & 258.09 & 37.89 & 41.59 & 42.45 & 257.54 & 40.72 & 43.26 & 44.17 \\
\hline
\end{tabular}

The achieved bit rates are given in kilobits per second, and the PSNR values for the luminance and the two chrominance components are given in decibels.

some of their key features can be obtained. For example, the MPEG-4 Simple profile provides approximately $15 \%$ bit-rate savings over H.263 Baseline. The technical features that should contribute to this improvement include allowing motion compensation on $8 \times 8$ blocks, extrapolation of motion vectors over picture boundaries, and improved intra coding efficiency. ${ }^{1}$ Additional bit-rate savings of $-7 \%$ to $14 \%$ are provided by H.263 CHC. The main technical difference between H.263 CHC and MPEG-4 SP is that H.263 CHC supports multiframe motion-compensated prediction and uses a modified chrominance quantization step size, which noticeably improves the chrominance fidelity ${ }^{2}$.

\section{Entertainment-Quality Applications}

Our third experiment seeks to address coding efficiency for entertainment-quality applications, such as DVD-Video systems and HDTV. In such applications, sequences are generally encoded at resolutions of $720 \times 480$ pixels and higher at average bit rates of $3 \mathrm{Mbit} / \mathrm{s}$ and up. Since the MPEG-2 standard is

\footnotetext{
${ }^{1}$ The maximum bit rate supported in any level of the Simple profile is only $384 \mathrm{kbits} / \mathrm{s}$ - a value that is exceeded by nearly every data point generated by the rate-distortion optimized encoder used in this test. Thus, only the simplest $30-\mathrm{Hz}$ CIF content can really be encoded with acceptable visual quality while conforming to the bit-rate restrictions of this profile. We have chosen to ignore this constraint in our analysis in order to measure the performance of the underlying technology rather than the confining the analysis only to cases within all limits of the MPEG-4 Visual specification.

${ }^{2}$ The rate-distortion as well as the bit-rate savings plots only consider the reconstruction quality of the luminance component.
}

the only standard commonly used in this application space, only its performance was compared to that of the H.264/AVC standard.

For this comparison we used a set of four interlaced-scan standard definition sequences at resolutions of $720 \times 576$ pixels $(25 \mathrm{~Hz})$ and four progressive-scan high-definition sequences at resolutions of $1280 \times 720$ pixels $(60 \mathrm{~Hz})$; details about these sequences are specified in Appendix B.

Aside from the higher resolution source content, the experimental setup is very similar to that used in the video streaming applications test. The same encoding software was used for both standards, as well as similar coding options, including two B-pictures between each pair of anchor pictures, Lagrangian coder control, and full-search motion estimation with a range of \pm 32 pixels. The MPEG-2 Visual encoder generated bitstreams that are compliant with the ML@MP and HL@MP conformance point for the standard definition and high-definition sequences, respectively. For H.264/AVC, the Main profile was used with five reference frames and CABAC as entropy coding. One key difference is that an I-picture was inserted every $480 \mathrm{~ms}$ for encoding the $25-\mathrm{Hz}$ standard definition sequences and every $500 \mathrm{~ms}$ for encoding the $60 \mathrm{-Hz}$ high-definition sequences. Frequent periodic INTRA coded pictures are typical in entertainment-quality applications in order to enable fast random access. As in the streaming test, the quantization parameter for B-pictures was set in a way that the resulting quantization step size is approximately $20 \%$ larger than that for P-pictures for both codecs. 

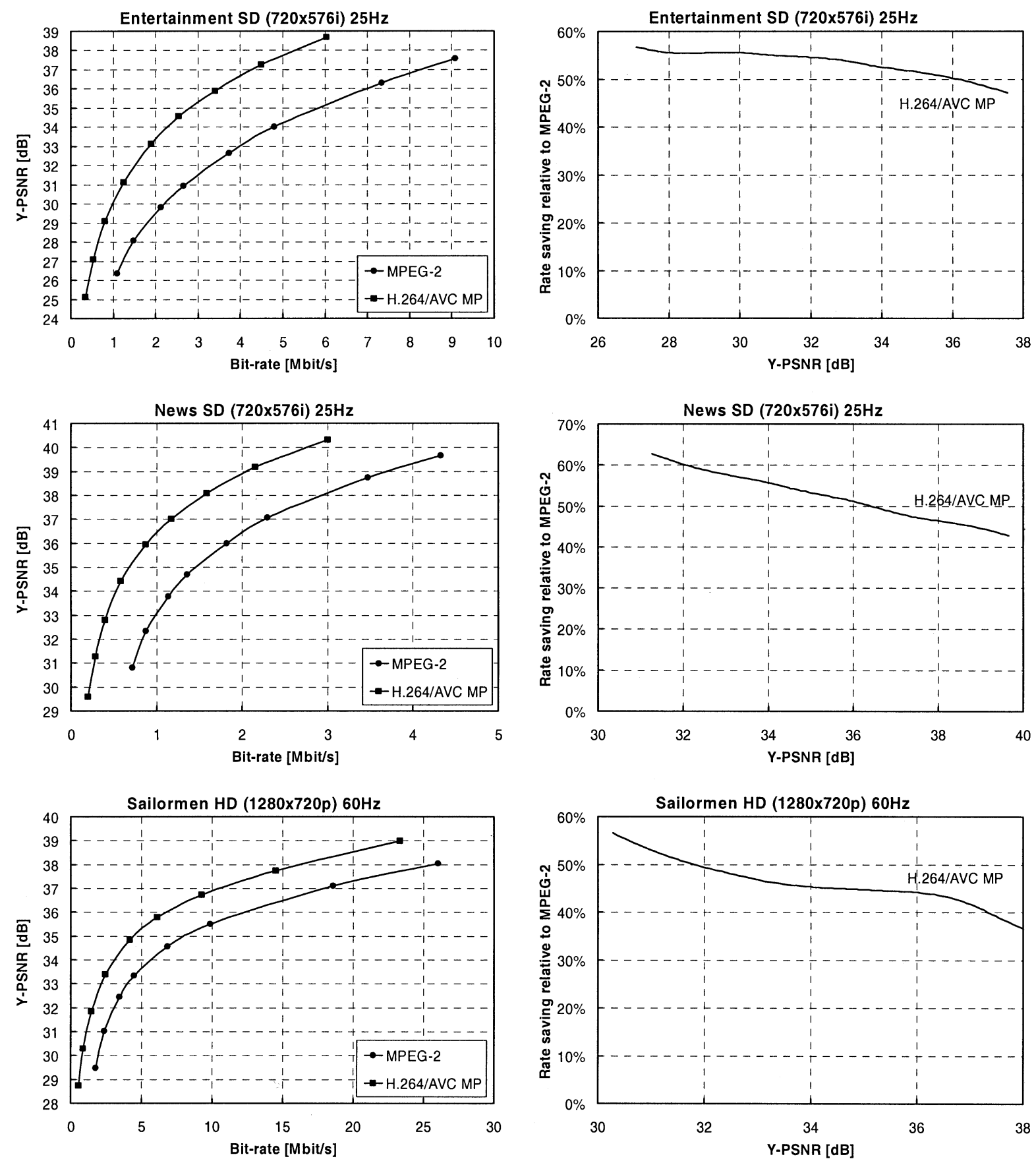

Fig. 3. Selected rate-distortion curve and bit-rate savings plot for entertainment-quality applications.

The rate-distortion curves generated by the two encoders, along with the computed bit-rate savings realized by H.264/AVC over MPEG-2 based on points of equal PSNR, are shown in Fig. 3 for three selected sequences. As in the previous tests, the H.264/AVC codec offers a significant rate savings advantage. At lower bit rates, savings lie between $45 \%$ and $65 \%$, while at the higher bit rates, which are more common in entertainment-quality applications, rate savings of $25 \%-45 \%$ are realized. The average rate saving measured over the entire set of sequences and bit-rates range is about $45 \%$.

\section{Subjective Comparisons}

While PSNR is a convenient measure of distortion in video applications, it does not take into account all of the intricacies of the human visual system, which is the ultimate judge of video quality. With this in mind, we have carried out a set of informal subjective visual tests in order to validate and complement the results that have been derived using PSNR-based comparison. The results of these tests indicate that H.264/AVC codec provides greater improvements in subjective quality over earlier standards than PSNR-based comparisons indicate.

One informal subjective test that was carried out at the HHI showed that for all cases in the streaming test set, sequences coded at $512 \mathrm{kbit} / \mathrm{s}$ with H.264/AVC are subjectively equivalent to the same sequences coded at $1024 \mathrm{kbit} / \mathrm{s}$ with MPEG-4 Visual. This corresponds to a bit-rate savings of H.264/AVC against MPEG-4 Visual of about 50\% for these bit rates, which is in general larger than the savings indicated on the rate-distor- 
TABLE V

INPUT SEQUENCES USED IN THE COMPARISON FOR VIDEO STREAMING APPLICATIONS

\begin{tabular}{l|l|l|l}
\hline Name & Res. & Duration & Characteristics \\
\hline Foreman & QCIF & $10 \mathrm{sec}$. & Fast camera and content motion with pan at the end \\
\hline Container Ship & QCIF & $10 \mathrm{sec}$. & Still camera on slow moving scene \\
\hline News & QCIF & $10 \mathrm{sec}$. & Still camera on human subjects with synthetic background \\
\hline Tempete & QCIF & $8.67 \mathrm{sec}$. & Camera zoom; spatial detail; fast random motion \\
\hline Bus & CIF & $5 \mathrm{sec}$. & Fast translational motion and camera panning; moderate spatial detail \\
\hline Flower Garden & CIF & $8.33 \mathrm{sec}$. & Slow and steady camera panning over landscape; spatial and color detail \\
\hline Mobile \& Calendar & CIF & $8.33 \mathrm{sec}$. & Slow panning and zooming; complex motion; high spatial and color detail \\
\hline Tempete & CIF & $8.67 \mathrm{sec.}$ & Camera zoom; spatial detail; fast random motion \\
\hline
\end{tabular}

tion plots. At lower bit rates, the tests seem to indicate bit-rate savings between $30 \%-40 \%$.

In a second set of informal subjective tests performed at UB Video, several H.264/AVC, MPEG-4 ASP, and H.263 CHC sequences with equivalent luminance PSNR were compared by a large number of viewers. Constant quantization parameters and no B-pictures were used by all encoders. The recommended de-blocking and de-ringing filters were applied as a post-process to the MPEG-4 ASP decoded sequences. The Test Model de-ringing filter was also applied to the H.263 CHC decoded sequences. Comparisons were made on each sequence between H.264/AVC and each of the other encoders, at both low and high bit rates. While each pair of sequences had nearly identical PSNR values, the test subjects indicated a significant preference for the H.264/AVC sequences relative to the MPEG-4 ASP sequences. The preference toward H.264/AVC was strongest on the low-bit-rate coded sequences. Again, these results indicate that the bit-rate savings that can be achieved using H.264/AVC to achieve essentially equivalent visual quality as other standards are even larger than what the PSNR-based results indicate. Finally, we note that in the H.264/AVC to H.263 CHC comparison, only a minor preference toward H.264/AVC was expressed, on average. The results of these tests suggest that the use of a de-blocking filter inside the motion compensation loop, as found in H.263 CHC and H.264/AVC but not MPEG-4 ASP, may have an impact on subjective visual quality beyond what is reflected in PSNR-based results.

Similar subjective comparisons were made between the MPEG-2 and H.264/AVC sequences encoded for the entertainment-quality test. Again, the results illustrated that the bit-rate savings that are provided by H.264/AVC are larger when subjective visual quality is used rather than PSNR measurements to determine points of equivalent quality. Approximately 10\%-15\% greater rate savings were observed for the H.264/AVC codec over a range of bit rates through subjective evaluation. At low bit rates, H.264/AVC was perceived to provide equivalent quality at a bit rate reduced by $70 \%$ from that of MPEG-2. At higher bit rates, rate savings of approximately $40 \%$ were determined based on the evaluation by the test subjects.

\section{CONCLUSIONS}

The performance of the H.264/AVC compliant encoder in all experiments clearly demonstrates the potential importance of this standard in future applications of video streaming as well as interactive video coding. Although H.264/AVC coding shares the common hybrid video coding structure with previous standards, there are significant differences that provide substantial coding gains. The main difference between H.264/AVC and most previous standards is the largely increased flexibility, which provides increased coding efficiency for potentially increased computational complexity at the encoder. This would require intelligent implementation and coder control strategies, especially in streaming and broadcast applications.

\section{APPENDIX I \\ VIDEO CODECS}

The software implementations used in the comparisons are as follows.

- MPEG-2: MPEG Software Simulation Group version 1.2. Public software, modified to include Lagrangian rate-distortion optimization. See http://www.mpeg.org/MSSG.

- H.263: University of British Columbia Signal Processing and Multimedia Group (UBC-SPMG), H.263 code library version 0.3. Available to ITU-T members and academic research organizations. See http://www.ece.ubc.ca/spmg/h263plus/h263plus.html.

- MPEG-4: The HHI MoMuSys-based rate-distortion optimized coder and the UB Video's UB-Stream version 2.0. Those two codecs were used to generate the anchors in MPEG's recent coding efficiency tests. See http://bs.hhi.de/ wiegand/ICG-Project-RDO.html and http://www.ubvideo.com.

- H.264/AVC: JVT JM-61e implementation developed by JVT members and with rate-distortion optimization by the HHI. Available at http://bs.hhi.de/ suehring/tml/download/jm61e.zip.

The various standard decoders together with bitstreams of all test cases presented in this paper can be downloaded at $\mathrm{ftp}: / / f t p . h h i . d e / i e e e-t c s v t /$.

\section{APPENDIX II} TEST SEQuENCES

Details about the input video sequences used in the comparisons for video streaming, videoconferencing, and entertainment applications are listed in Tables V-VII, respectively. All sequences use the YUV 4:2:0 color format, in which 
TABLE VI

INPUT SEQUENCES USED IN THE COMPARISON FOR VIDEOCONFERENCING APPLICATIONS

\begin{tabular}{l|l|l|l}
\hline Name & Res. & Duration & Characteristics \\
\hline Akiyo & QCIF & $10 \mathrm{sec}$. & Still camera on human subject with synthetic background \\
\hline Foreman & QCIF & $10 \mathrm{sec}$. & Fast camera and content motion with pan at the end \\
\hline Silent & QCIF & $10 \mathrm{sec}$. & Still camera but fast moving subject \\
\hline Mother \& Daughter & QCIF & $10 \mathrm{sec}$. & Still camera on human subjects \\
\hline Carphone & CIF & $10 \mathrm{sec}$. & Fast camera and content motion with landscape passing \\
\hline Foreman & CIF & $10 \mathrm{sec}$. & Fast camera and content motion with pan at the end \\
\hline Paris & CIF & $10 \mathrm{sec}$. & Still camera on human subjects; typical videoconferencing content \\
\hline Sean & CIF & $10 \mathrm{sec}$. & Still camera on human subject with synthetic background \\
\hline
\end{tabular}

TABLE VII

INPUT SEQUENCES USED IN THE COMPARISON FOR ENTERTAINMENT APPLICATIONS

\begin{tabular}{l|l|l|l}
\hline Name & Res. & Duration & Characteristics \\
\hline Harp \& Piano & $720 \times 576 \mathrm{i}$ & $8.8 \mathrm{sec}$. & Fast camera zoom; local motion \\
\hline Basketball & $720 \times 576 \mathrm{i}$ & $9.92 \mathrm{sec}$. & Fast camera and content motion; high spatial detail \\
\hline Entertainment & $720 \times 576 \mathrm{i}$ & $10 \mathrm{sec}$. & Camera and content motion; spatial detail \\
\hline News & $720 \times 576 \mathrm{i}$ & $10 \mathrm{sec}$. & Scene cut between slow and fast moving scene \\
\hline Shuttle Start & $1280 \times 720 \mathrm{p}$ & $10 \mathrm{sec}$. & Jiggling camera, low contrast, lighting change \\
\hline Sailormen & $1280 \times 720 \mathrm{p}$ & $10 \mathrm{sec}$. & Translational and random motion; high spatial detail \\
\hline Night & $1280 \times 720 \mathrm{p}$ & $7.67 \mathrm{sec}$. & Static camera, fast complex motion \\
\hline Preakness & $1280 \times 720 \mathrm{p}$ & $10 \mathrm{sec}$. & Camera zoom, highly complex motion, high spatial detail \\
\hline
\end{tabular}

the two chrominance components are down-sampled by a factor of two in each spatial direction. The sequences used in the first two comparisons are popular QCIF and CIF resolution test sequences used in the video standards community.

\section{APPENDIX III \\ PERFORMANCE MEASURES}

Since it is the most widely accepted objective measure of visual distortion, PSNR of the luminance component is our primary means of measuring visual distortion. The PSNR between the reconstructed $\left(s^{\prime}\right)$ and the original $(s)$ video signal for the set of pixels in $\boldsymbol{A}$ is determined via

$$
\text { PSNR }=10 \log _{10} \frac{255^{2}}{\mathrm{MSE}} \mathrm{dB}
$$

with

$$
\mathrm{MSE}=\frac{1}{|\mathbf{A}|} \mathrm{SSD}
$$

where the SSD is given via (6) and $|\boldsymbol{A}|$ specifies the number of pixels in $\boldsymbol{A}$.

For each test case and sequence, results are presented in a set of rate-distortion curves, with one curve for each encoder being evaluated. A curve is generated by encoding each sequence several times with different quantization step sizes, which are held constant throughout each of the coding passes. The average PSNR for each of the three components over all of the frames in the sequence is recorded and plotted versus the average bit rate. These results indicate differences in achievable rate-distortion performance between different standards.
A more practical and tangible quantity to measure is the percentage bit-rate savings that one standard can provide relative to another, while achieving equivalent visual quality. These calculations can be made by interpolating between points on two rate-distortion curves, aligning points of equal distortion, and then computing the difference in bit rate between these points. In order to make such comparisons between several rate-distortion curves, the curve of the encoder with the poorest performance is used as a common base for comparison against all of the other encoders. This can be expressed as

$$
S(\mathrm{PSNR})=100 \cdot \frac{A(\mathrm{PSNR})-B(\mathrm{PSNR})}{A(\mathrm{PSNR})} \%
$$

where $B$ and $A$ represent the bit rates necessary to achieve a given PSNR value, using the encoder in question $(B)$ and the common anchor encoder $(A)$, respectively.

While these objective measures are convenient and widely accepted, we recognize that the ultimate judge of quality is the human viewer. To this end, small-scale informal subjective tests were conducted in order to validate the results found using PSNR measures. Sequences used in the tests achieved a target bit rate, within a tolerance of $\pm 2 \%$ by selecting the necessary fixed quantizer to achieve the rate. One change in the quantizer value was permitted at some point in the sequence, to facilitate meeting the target rate within the small tolerance.

\section{ACKNOWLEDGMENT}

The authors would like to thank the JVT for the collaborative work and the technically outstanding discussions and contributions that enabled this analysis. 


\section{REFERENCES}

[1] "Generic Coding of Moving Pictures and Associated Audio Information - Part 2: Video," ITU-T and ISO/IEC JTC1, ITU-T Recommendation H.262-ISO/IEC 13 818-2 (MPEG-2), 1994

[2] "Video Coding for Low Bitrate Communication Version 1," ITU-T, ITU-T Recommendation H.263, 1995.

[3] "Coding of Audio-Visual Objects-Part 2: Visual," ISO/IEC JTC1, ISO/IEC 14 496-2 (MPEG-4 visual version 1), 1999.

[4] T. Wiegand, G. J. Sullivan, and A. Luthra, "Draft ITU-T Recommendation H.264 and Final Draft International Standard 14496-10 Advanced Video Coding," Joint Video Team of ISO/IEC JTC1/SC29/WG11 and ITU-T SG16/Q.6, Doc. JVT-G050rl, Geneva, Switzerland, May 2003.

[5] T. Wiegand and B. D. Andrews, "An Improved H.263 Coder Using RateDistortion Optimization,”, Tampere, Finland, ITU-T/SG16/Q15-D-13, 1998.

[6] M. Gallant, G. Cote, and F. Kossentini, "Description of and Results for Rate-Distortion Based Coder,", Tampere, Finland, ITU-T/SG16/Q15-D-47, 1998

[7] "Video Codec Test Model Near-Term Number 10 (TMN-10),", Tampere, Finland, ITU-T/SG 16/VCEG (formerly Q.15, now Q.6), 1998.

[8] "Working draft number 2, revision 8 (WD-2 rev 8)," in Joint Video Team of ISO/IEC MPEG and ITU-T VCEG, JVT-B118r8, T. Wieg, Ed., Apr. 2002.

[9] "MPEG-4 Video Verification Model 18.0 (VM-18)," ISO/IEC JTC1/SC29/WG11, Doc. MPEG-N3908, 2001.

[10] “Test Model 5," ISO/IEC JTC1/SC29/WG11, Doc. MPEG-N0400, 1993.

[11] "Video Codec for Audiovisual Services at $\mathrm{p} \times 64 \mathrm{Kbit} / \mathrm{s}$ Version 1," ITU-T, ITU-T Recommendation H.261, 1990.

[12] J. L. Mitchell, W. B. Pennebaker, C. Fogg, and D. J. LeGall, MPEG Video Compression Standard. New York: Chapman \& Hall, 1997.

[13] B. G. Haskell, A. Puri, and A. N. Netravalli, Digital Video: An Introduction to MPEG-2. New York: Chapman \& Hall, 1997.

[14] "Coding of Moving Pictures and Associated Audio for Digital Storage Media at up to About $1.5 \mathrm{Mbit} / \mathrm{s}$-Part 2: Video," ISO/IEC JTC1, ISO/IEC 11 172-2 (MPEG-1), 1993.

[15] G. Côté, B. Erol, M. Gallant, and F. Kossentini, "H.263+: Video coding at low bit rates," IEEE Trans. Circuits Syst. Video Technol., vol. 8, pp. 849-866, Nov. 1998

[16] T. Wiegand, G. J. Sullivan, G. Bjøntegaard, and A. Luthra, "Overview of the H.264/AVC video coding standard"," IEEE Trans. Circuits Syst. Video Technol., vol. 13, pp. 560-576, July 2003.

[17] M. Flierl and B. Girod, "Generalized B pictures and the draft H.264/AVC video compression standard"," IEEE Trans. Circuits Syst. Video Technol., vol. 13, pp. 587-597, July 2003.

[18] D. Marpe, H. Schwarz, and T. Wiegand, "Context-adaptive binary arithmetic coding for H.264/AVC," IEEE Trans. Circuits Syst. Video Technol., vol. 13, pp. 620-636, July 2003.

[19] T. Stockhammer, M. M. Hannuksela, and T. Wiegand, "H.264/AVC in wireless environments," IEEE Trans. Circuits Syst. Video Technol., vol. 13 , pp. 657-673, July 2003.

[20] H. Everett, "Generalized lagrange multiplier method for solving problems of optimum allocation of resources," Oper. Res., vol. 11, pp. 399-417, 1963

[21] Y. Shoham and A. Gersho, "Efficient bit allocation for an arbitrary set of quantizers," IEEE Trans. Acoust., Speech, Signal Processing, vol. 36, pp. 1445-1453, Sept. 1988.

[22] T. Wiegand, X. Zhang, and B. Girod, "Long-term memory motion-compensated prediction," IEEE Trans. Circuits Syst. Video Technol., vol. 9, pp. 70-84, Feb. 1999.

[23] T. Wiegand and B. Girod, Multi-Frame Motion-Compensated Prediction for Video Transmission. Norwell, MA: Kluwer, 2001.

[24] G. J. Sullivan and T. Wiegand, "Rate-distortion optimization for video compression," IEEE Signal Processing Mag., vol. 15, pp. 74-90, Nov. 1998.

[25] G. J. Sullivan and R. L. Baker, "Rate-distortion optimized motion compensation for video compression using fixed or variable size blocks," in Proc. GLOBECOM'91, Phoenix, AZ, Dec. 1991, pp. 85-90.

[26] T. Wiegand, M. Lightstone, D. Mukherjee, T. G. Campbell, and S. K. Mitra, "Rate-distortion optimized mode selection for very low bit rate video coding and the emerging H.263 standard," IEEE Trans. Circuits Syst. Video Technol., vol. 6, pp. 182-190, Apr. 1996.

[27] T. Wiegand and B. Girod, "Lagrangian multiplier selection in hybrid video coder control," in Proc. ICIP 2001, Thessaloniki, Greece, Oct. 2001.

[28] "Call for Proposals on New Tools for Video Compression Technology," ISO/IEC JTC1/SC29/WG11, Doc. MPEG-N4065, 2001.

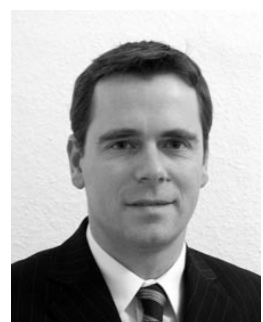

Thomas Wiegand received the Dr.-Ing. degree from the University of Erlangen-Nuremberg, Germany, in 2000 and the Dipl.-Ing. degree in electrical engineering from the Technical University of Hamburg-Harburg, Germany, in 1995

$\mathrm{He}$ is the Head of the Image Communication Group in the Image Processing Department, Fraunhofer-Institute for Telecommunications - Heinrich Hertz Institute (HHI), Berlin, Germany. During 1997 to 1998 , he was a Visiting Researcher at Stanford University, Stanford, CA, and served as a Consultant to 8x8, Inc., Santa Clara, CA. From 1993 to 1994, he was a Visiting Researcher at Kobe University, Kobe, Japan. In 1995, he was a Visiting Scholar at the University of California at Santa Barbara, where he began his research on video compression and transmission. Since then, he has published several conference and journal papers on the subject and has contributed successfully to the ITU-T Video Coding Experts Group (ITU-T SG16 Q.6-VCEG)/ISO/IEC Moving Pictures Experts Group (ISO/IEC JTC1/SC29/WG11-MPEG)/Joint Video Team (JVT) standardization efforts and holds various international patents in this field. He has been appointed as the Associated Rapporteur of the ITU-T VCEG (October 2000), the Associated Rapporteur/Co-Chair of the JVT that has been created by ITU-T VCEG and ISO/IEC MPEG for finalization of the H.264/AVC video coding standard (December 2001), and the Editor of the H.264/AVC video coding standard (February 2002)

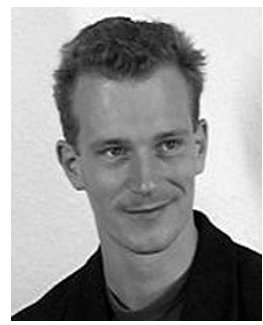

Heiko Schwarz received the Dipl.-Ing. degree in electrical engineering in 1996 and the Dr.-Ing. degree in 2000, both from the University of Rostock, Rostock, Germany.

In 1999, he joined the Fraunhofer-Institute for Communications, HHI, Berlin, Germany. Since then, he has contributed successfully to the ITU-T Video Coding Experts Group (VCEG) and the Joint Video Team (JVT) standardization efforts. His research interests include image and video compression, video communication as well as signal processing

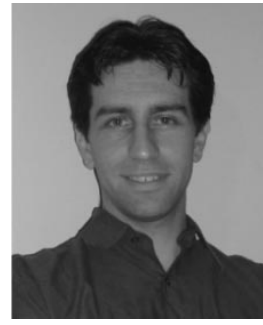

Anthony Joch received the B.Eng. degree in computer engineering from McMaster University, Hamilton, ON, Canada, in 1999, and the M.A.Sc. degree in electrical engineering from the University of British Columbia, Vancouver, BC, Canada, in 2002.

In 2000, he joined UB Video Inc., Vancouver, $\mathrm{BC}$, where he is currently a Senior Engineer involved in the development of software codecs for the H.264/MPEG-4 AVC standard. His research interests include reduced-complexity algorithms for video encoding, video pre- and post-processing, and multimedia systems. He has been an active contributor to the H.264/MPEG-4 AVC standardization effort, particularly in the area of deblocking filtering and as a co-chair of the ad-hoc group for bitstream exchange.

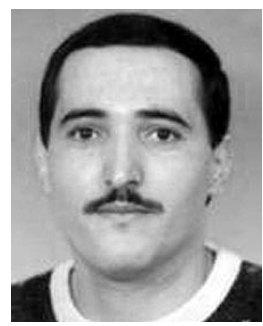

Faouzi Kossentini (S'89-M'95-SM'98) received the B.S., M.S., and Ph.D. degrees from the Georgia Institute of Technology, Atlanta, in 1989, 1990, and 1994, respectively.

$\mathrm{He}$ is presently the President and Chief Executive Officer of UB Video, Vancouver, BC, Canada. He is also an Associate Professor in the Department of Electrical and Computer Engineering, University of British Columbia, Vancouver, BC, Canada, performing research in the areas of signal processing, communications, and multimedia. He has co-authored over 150 journal papers, conference papers, and book chapters. He has also participated in numerous international ISO and ITU-T activities involving the standardization of coded representation of audiovisual information.

Dr. Kossentini was an Associate Editor for the IEEE TRANSACTIONS ON ImAGE PROCESSING and the IEEE TRANSACTIONS ON Multimedia and a Vice General Chair for ICIP-2000. 


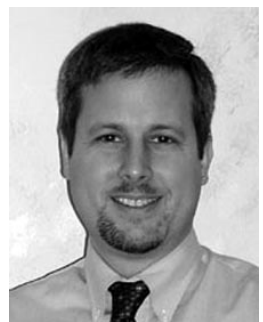

Gary J. Sullivan (S'83-M' 91-SM'01) received the B.S. and M.Eng. degrees in electrical engineering from the University of Louisville, Louisville, KY, in 1982 and 1983, respectively, and the Ph.D. and Eng. degrees in electrical engineering from the University of California, Los Angeles, in 1991.

$\mathrm{He}$ is the Chairman of the Joint Video Team (JVT) for the development of the next-generation H.264/MPEG4-AVC video coding standard, which was recently completed as a result of a joint project between the ITU-T video coding experts group (VCEG) and the ISO/IEC moving picture experts group (MPEG). He is also the Rapporteur of Advanced Video Coding in the ITU-T, where he has led VCEG (ITU-T Q.6/SG16) for about six years, and the ITU-T Video Liaison Representative to MPEG (ISO/IEC JTC1/SC29/WG11) and served as MPEG's Video Chairman during 2001-2002. He is currently a Program Manager of video standards and technologies in the eHome A/V Platforms Group of Microsoft Corporation, Redmond, WA, where he designed and remains lead engineer for the DirectX ${ }^{\circledR}$ Video Acceleration API/DDI feature of the Microsoft Windows ${ }^{\circledR}$ operating system platform. Prior to joining Microsoft in 1999, he was the Manager of Communications Core Research at PictureTe Corporation, the quondam world leader in videoconferencing communication He was previously a Howard Hughes Fellow and Member of the Technical Staff in the Advanced Systems Division of Hughes Aircraft Corporation and was a terrain-following radar system software engineer for Texas Instruments. His research interests and areas of publication include image and video compression, rate-distortion optimization, motion representation, scalar and vector quantization, and error- and packet-loss-resilient video coding. 